\title{
Detection of Polyps Including Adenomas in the Ascending Colon by Cap-assisted Chromocolonoscopy with Indigo Carmine
}

\author{
Hyun Seok Lee \\ Department of Internal Medicine, Kyungpook National University Medical Center, Kyungpook National University School of Medicine, Daegu, \\ Korea
}
Article: The Effect of Indigocarmine on Improvement of the Polyp Detection Rate during Colonoscopic Examination with Hood Cap (Intest Res 2014;12:60-65)

Adenomatous polyps are precursors of colorectal cancer, and their removal is important because it could potentially reduce the incidence and mortality of colorectal cancer. These concepts have formed the basis for the removal of adenomatous polyps after detection. ${ }^{1}$ Nonpolypoid adenomas, which are at an increased risk of developing into colorectal cancer, are difficult to detect during routine colonoscopy, and small adenomas may be missed during colonoscopy. ${ }^{2}$

Cap-assisted colonoscopy and chromocolonoscopy have emerged as techniques with the potential to improve the detection of colon polyps. Cap-assisted colonoscopy uses a transparent plastic hood attached to the tip of the colonoscope to flatten the semilunar folds and improve mucosal exposure. $^{3}$ A systemic research and meta-analysis of 12 studies that have examined the usefulness of cap-assisted colonoscopy for polyp detection found that cap-assisted colonoscopy was associated with improved detection of colorectal polyps. ${ }^{3}$

Chromocolonoscopy is a colonoscopic examination performed using the indigo carmine spray technique. Togashi et al. ${ }^{4}$ described that chromocolonoscopy increased the detection of neoplastic polyps and flat adenomas, particularly diminutive polyps, but did not increase the detection of advanced lesions. ${ }^{4}$ Hashimoto et al. ${ }^{5}$ showed that the adenomatous polyp detection rate of chromocolonoscopy was

Received November 29, 2013. Revised December 5, 2013.

Accepted December 5, 2013.

Correspondence to Hyun Seok Lee, Department of Internal Medicine, Kyungpook National University Medical Center, Kyungpook National

University School of Medicine, 807 Hoguk-ro, Buk-gu, Daegu 702-210, Korea. Tel: +82-53-200-2603, Fax: +82-53-200-2027, E-mail: Ihsworld@nate.com

Financial support: None. Conflict of interest: None. not better than that of standard colonoscopy. Thus, whether chromocolonoscopy using indigo carmine increases the detection of colorectal polyps remains controversial. ${ }^{4,5}$

Additional indigo carmine spraying during cap-assisted colonoscopy is expected to improve the detection rate of colon polyps, especially adenomatous polyps. Therefore, this study aimed to evaluate the usefulness of cap-assisted chromocolonoscopy in the proximal colon for routine colonoscopic examinations. ${ }^{6}$ Hood cap-assisted colonoscopic examination was first performed, followed by hood capassisted chromocolonoscopy with $0.2 \%$ indigo carmine, from the cecum to the hepatic flexure. The total number and characteristics of polyps were compared before and after spraying indigo carmine.

Cap-assisted chromocolonoscopy was performed in 86 consecutive patients. Before indigo carmine spraying, a mean of 0.56 polyps per patient and 0.29 adenomas per patient ( $52 \%$ of the polyps) were found. After spraying of the dye, a mean of 0.62 polyps and 0.22 adenomas (36\% of these additional polyps) were found additionally. Most (85\%) of these additional polyps found after spraying were small $(<0.5 \mathrm{~cm})$. In total, a mean of 1.17 polyps and 0.51 adenomas ( $44 \%$ of total polyps) were found before and after spraying of the dye. In this study, hood cap-assisted chromocolonoscopy using indigo carmine showed a higher detection rate of polyps (especially, small polyps $<0.5 \mathrm{~cm}$ ) and adenomas in the ascending colon than cap-assisted colonoscopy without spraying of the dye.

It is unclear whether cap-assisted chromocolonoscopy can increase the detection rate of polyps and adenomas compared with chromocolonoscopy without cap.

๑ Copyright 2014. Korean Association for the Study of Intestinal Diseases. All rights reserved.

This is an Open Access article distributed under the terms of the Creative Commons Attribution Non-Commercial License (http://creativecommons.org/licenses/by-nc/3.0)

which permits unrestricted non-commercial use, distribution, and reproduction in any medium, provided the original work is properly cited. 
This study has some limitations. It was a single-institution analysis, and a limited number of patients were included. Because only one laterally spreading tumor and one polyp $>1$ $\mathrm{cm}$ were observed in this study, the detection rate of laterally spreading tumors or large polyps could not be analyzed.

The clinical significance of these small adenomas that can be found only after spraying a contrast agent needs to be further investigated. ${ }^{2}$

\section{REFERENCES}

1. Winawer SJ, Zauber AG, O'Brien MJ, et al. Randomized comparison of surveillance intervals after colonoscopic removal of newly diagnosed adenomatous polyps. The National Polyp Study Workgroup. N Engl J Med 1993;328:901-906.

2. Lee JH, Kim JW, Cho YK, et al. Detection of colorectal adenomas by routine chromoendoscopy with indigocarmine. Am J Gastroenterol 2003;98:1284-1288.

3. Westwood DA, Alexakis N, Connor SJ. Transparent cap-assisted colonoscopy versus standard adult colonoscopy: a systematic review and meta-analysis. Dis Colon Rectum 2012;55:218-225.

4. Togashi K, Hewett DG, Radford-Smith GL, Francis L, Leggett BA, Appleyard MN. The use of indigocarmine spray increases the colonoscopic detection rate of adenomas. J Gastroenterol 2009; 44:826-833.

5. Hashimoto K, Higaki S, Nishiahi M, Fujiwara K, Gondo T, Sakaida I. Does chromoendoscopy improve the colonoscopic adenoma detection rate? Hepatogastroenterology 2010;57:13991404 .

6. Kwon SC, Choi SW, Choi SH, et al. The effect of indigocarmine on improvement of the polyp detection rate during colonoscopic examination with hood cap. Intest Res 2014;12:60-65. 